index ecomunicación | no 10(1) 2020 | Páginas 219-240

E-ISSN: 2174-1859 | ISSN: 2444-3239 | Depósito Legal: M-19965-2015

Recibido el 13_04_2020 | Aceptado el 13_09_2020 | Publicado el 15_10_2020

\title{
DEL ESPECTADOR PASIVO AL EXPERIENCIAL: LA REALIDAD VIRTUAL EN LA INFORMACIÓN DEPORTIVA
}

FROM PASSIVE TO EXPERIENTIAL SPECTATOR: VIRTUAL REALITY IN SPORTS INFORMATION

https://doi.org/10.33732/ixc/10/01Delesp

Adriana Paíno-Ambrosio

Universidad Europea Miguel de Cervantes apaino@uemc.es https://orcid.org/0000-0003-3817-6071

M. a Isabel Rodríguez-Fidalgo Universidad de Salamanca mrfidalgo@usal.es https://orcid.org/0000-0001-5167-0272 
Resumen: La Realidad Virtual (RV) y el vídeo en $360^{\circ}$ se han introducido de forma novedosa en los últimos años en el ámbito periodístico gracias a sus potencialidades narrativas. Sus características no han pasado desapercibidas dentro del campo de la información deportiva, que ya cuenta con una elevada audiencia en cualquier medio de comunicación tradicional o cibernético, dotándola, ahora, de un mayor atractivo. Uno de los cambios que introducen estas producciones es su forma de consumo. Dentro de este contexto surge la presente investigación, centrada en el periodismo inmersivo. Bajo una perspectiva cualitativa y cuantitativa se analizan 225 producciones deportivas seleccionadas aleatoriamente en las principales plataformas técnicas que suministran contenidos en RV y vídeo en $360^{\circ}$. La perspectiva del análisis se centra en los cambios producidos en el espectador deportivo. Los principales hallazgos evidencian que una de las innovaciones de este tipo de narración inmersiva incide directamente en el rol que ocupa el espectador, al dejar de adoptar una actitud pasiva para convertirse en un protagonista más del deporte. Comienzan a generarse nuevas experiencias de consumo deportivas donde el tratamiento informativo, en cierta medida, queda relegado a un segundo plano, en relación a los criterios periodísticos tradicionales. Estamos ante un nuevo espectador deportivo.

Palabras clave: Realidad Virtual; vídeo en $360^{\circ}$; deportes; espectador deportivo; inmersión; experiencial.

Abstract: Virtual Reality (VR) and $360^{\circ}$ video have been introduced in a novel way in recent years in the journalistic field thanks to their narrative potentialities. Their characteristics have not gone unnoticed within the field of sports reporting, which already has a high audience in any traditional or cybernetic media, giving it a greater attraction now. One of the changes introduced by these productions is their form of consumption. Within this context, this research arises, focused on immersive journalism. Under a qualitative and quantitative perspective, 225 sports productions randomly selected from the main technical platforms that provide VR and video content in $360^{\circ}$ are analyzed. The perspective of the analysis focuses on the changes produced in the sports viewer. The main findings show that one of the innovations of this type of immersive storytelling directly affects the role of the spectator, who no longer adopts a passive attitude but becomes a protagonist of the sport. New experiences of sports consumption are beginning to be generated where the treatment of information, to a certain extent, is 
relegated to second place, in relation to traditional journalistic criteria. We are in front of a new sports spectator.

Keywords: Virtual reality; $360^{\circ}$ video; Sports; Sports Spectator; Immersion; Experiential.

\section{Introducción}

El mundo de la comunicación atraviesa un momento de importantes transformaciones que se vinculan a la utilización de las nuevas tecnologías digitales. En poco tiempo, los medios de comunicación han cambiado sus rutinas a la hora de producir la información, lo que ha llevado a que los periodistas deban reactualizarse constantemente (Nunes y Canavilhas, 2020; Salaverria, 2020). Las nuevas tecnologías están dando lugar a la aparición de contenidos realizados bajo narrativas que no habían sido utilizadas hasta ahora, donde prima especialmente la participación de la audiencia. Estas innovaciones, por lo tanto, no se centran únicamente en una adquisición tecnológica por parte de las empresas de comunicación, sino también en una formación de los propios profesionales de la información, tanto a la hora de utilizar dichos equipamientos, como de proponer nuevos contenidos que se ajusten a las demandas de un usuario cada vez más activo dentro de la comunicación digital.

La información deportiva ha sabido adaptarse a todos estos cambios que se han ido produciendo. La elevada audiencia que mueve el deporte, con las retransmisiones de los eventos deportivos, financiados con grandes inversiones publicitarias, ha propiciado que la comunicación deportiva sea puntera en la utilización de nuevas tecnologías y narrativas (Herrero, 2019). Prueba de ello es que, cada vez más, se estén utilizando en dichas coberturas informativas las últimas tecnologías en llegar, como la Realidad Virtual (RV) y el vídeo en $360^{\circ}$. Medios de comunicación en todo el mundo cuentan ya, en mayor o menor medida, con producciones inmersivas con el objetivo de informar de estos eventos desde un punto de vista novedoso y atractivo para conseguir un mayor índice de audiencia.

La RV y el vídeo en $360^{\circ}$ introducen, como principal novedad, que el espectador-usuario accede a los contenidos en primera persona, no en tercera como sucedía en la información tradicional. Las recreaciones tridimensionales y las grabaciones omnidireccionales sobre los encuentros deportivos, además de cumplir con la función periodística de informar, abordan también una perspectiva lúdica o de entretenimiento centrada en la experiencia del usuario que está consumiendo esa información deportiva (Sidorenko, Cantero y He- 
rranz, 2018). Esto está dando lugar a contenidos que hacen posible, por ejemplo, que el espectador pueda ser un jugador más en un partido de fútbol, copiloto en un rally o colocarse sobre los esquíes de un esquiador profesional; véase, por ejemplo, Ski with Ted Ligety (USA Today, 2016). Precisamente es en este punto donde se asienta la investigación que aquí se plantea, la cual se centra en el denominado periodismo inmersivo y hace hincapié en explorar los nuevos roles que adopta el nuevo espectador deportivo.

\section{Marco teórico}

El estudio planteado se articula en torno a tres ejes desde el punto de vista teórico. El primero de ellos hace alusión al periodismo que utiliza la RV y el vídeo en $360^{\circ}$ para crear contenidos informativos, conocido como periodismo inmersivo. Relacionado directamente con él se encuentra el segundo eje referido al concepto de 'presencia', que se centra en la perspectiva que adopta el usuario a la hora de consumir una producción inmersiva. Por último, es necesario abordar también cómo se está aplicando el periodismo inmersivo dentro de la comunicación deportiva en los últimos años, puesto que son muchos los productores que apuestan por su utilización en dicho ámbito.

\subsection{El periodismo inmersivo: concepto e innovaciones}

Hoy en día, la utilización del término Realidad Virtual se ha popularizado y ha dejado de ser un invento asociado únicamente a la ciencia ficción. Se ha producido un cambio sustancial y una tecnología que, hace apenas unos años, era todavía muy desconocida ha pasado a ser un reclamo en la sociedad. La industria del entretenimiento y, especialmente, de los videojuegos ha contribuido a su popularización. Efectivamente, los juegos en RV son una de las principales vías de acceso de los usuarios a los contenidos inmersivos. Si bien es cierto, el abaratamiento de sus dispositivos, especialmente de los aparatos de visualización que hacen posible su consumo - gafas de RV- y el hecho de que las grandes compañías tecnológicas - como Oculus, Samsung o HTC, entre otrasapuesten fuertemente por su desarrollo, han sido clave para que la RV se haya convertido en un producto muy atractivo, tanto por parte de los productores como de los consumidores. Según los datos aportados por la consultora International Data Corporation (2019) se calcula que en 2020 el gasto mundial en realidad virtual y aumentada (RV/RA) será de 18.800 millones de dólares, lo que supone un incremento del 78,5\% con respecto al 2019.

Ahora bien, ¿qué ofrece la RV? Para ello es necesario remitirse a su definición. Son muchos los autores que, desde hace tiempo, vienen aportando definiciones sobre dicho concepto, como Biocca y Levy, 1995; Brudniy y Demilhanova, 2012; Burdea y Coiffet, 2003; Gubern, 1996; Lanier, 1989; o Sher- 
man y Craig, 2003, entre otros. Todos ellos comparten que la RV está ligada a un universo generado a través de un software con el objetivo de crear un espacio que el usuario percibe como real. De este modo, en palabras de Lanier

It recreates our relationship with the physical world in a new plane, no more, no less. It doesn't affect the subjective world; it doesn't have anything to do directly with what's going on inside your brain. It only has to do with what your sense organs perceive (Lanier, 1989: 110) ${ }^{1}$.

A medida que se ha avanzado técnicamente han ido surgiendo otros términos que se asocian a la RV, es el caso del vídeo en 360․ Este último ha adquirido un especial protagonismo a raíz del desarrollo de las cámaras que realizan grabaciones omnidireccionales, a través de dos o más lentes, y cuyo resultado final ofrece una imagen en $360^{\circ}$. Este tipo de grabaciones comparte con la RV que pueden ser consumidas de manera inmersiva con gafas de RV. No hay que olvidar que el vídeo en $360^{\circ}$ presenta ciertas limitaciones en relación a la RV, que afectan directamente a la libertad de movimiento del usuario, el cual no se puede mover dentro de la grabación efectuada y donde adquiere la posición que ocupa la cámara (Slater y Sánchez-Vives, 2016). Hoy en día, no cesan los avances técnicos en este campo y son muchas las piezas inmersivas que combinan una parte de recreación tridimensional, generada por ordenador, con imágenes reales capturadas en $360^{\circ}$.

Teniendo en cuenta estos aspectos, hay que mencionar las principales innovaciones que han introducido este tipo de tecnologías y que están ligadas a la narración inmersiva. Estas permiten al usuario asistir en primera persona a los hechos narrados, a través de unas imágenes que recogen la totalidad del espacio, y ya no está limitada al tipo de encuadre que ofrece la imagen audiovisual tradicional. Por esta razón, el clásico montaje se redefine al participar también el usuario, ya que puede ir seleccionando el fragmento de la realidad que quiere ver dentro de este todo registrado. Además, el tratamiento del sonido adquiere una nueva dimensión al estar relacionado directamente con un mayor nivel de inmersión del usuario.

Estos avances narrativos han sido muy bien aprovechados por el ámbito periodístico que no ha dudado en incluirlos en sus prácticas a la hora de contar la realidad. La pionera dentro de este campo es Nonny de la Peña, a la que se atribuye el término periodismo inmersivo, que es entendido por esta autora como «la producción de noticias en una forma que permite a las personas ob-

\footnotetext{
${ }^{1}$ Recrea nuestra relación con el mundo físico en un nuevo plano, ni más ni menos. No afecta al mundo subjetivo; no tiene nada que ver directamente con lo que está pasando dentro de tu cerebro. Sólo tiene que ver con lo que perciben tus órganos sensoriales. Traducción por parte de las autoras.
} 
tener experiencias en primera persona sobre eventos o situaciones descritas en las historias periodísticas» (de la Peña et al. 2010: 291).

Diez años después de esta definición, los medios de comunicación a nivel mundial ofrecen ya - aunque no de manera generalizada - este tipo de producciones inmersivas entre sus contenidos. Esta utilización de la RV y el vídeo en $360^{\circ}$ para abordar los contenidos informativos ha sido también objeto de interés en la literatura científica (Benítez y Herrera, 2017; Bujic y Amari, 2020; Colussi y Reis, 2020; Kang et al., 2019; Paíno y Rodríguez, 2019; PérezSeijo y López-García, 2019; Santos, 2019).

\subsection{Los conceptos de inmersión, presencia e interactividad vinculados a la RV y el vídeo en $360^{\circ}$}

Para hablar de tecnologías inmersivas es preciso abordar tres conceptos: inmersión, presencia e interactividad que, si bien se utilizan de manera conjunta, tienen connotaciones diferentes. El primero de ellos alude a un aspecto objetivo que se vincula a los estímulos sensoriales que vienen dados por la tecnología, de modo que cuanto más fieles sean dichos estímulos a sus equivalentes en el mundo real, mayor será el grado de inmersión.

Por su parte, el concepto de presencia alude a la respuesta del usuario a esos estímulos y hace referencia al grado en el que dichos usuarios tienen la sensación de encontrarse en un lugar diferente al de su ubicación real (Heeter, 1992; Nash, 2018; Shim y Jounghuyu, 2003; Slater, 1999, 2003; Slater, Lotto, Arnold, Sánchez-Vives, 2009; Witmer y Singer, 1994, 1998).

La interactividad es otro de los factores directamente relacionados con las producciones inmersivas, pero no a todas, sólo a aquellas en las que el entorno virtual proporciona al usuario la posibilidad de llevar a cabo acciones dentro del espacio virtual diseñado. De este modo, si entre el usuario y el medio se produce una interacción fluida el grado de presencia será mayor; pero si, por el contrario, se produce algún problema a la hora de realizar esa interacción esto revertirá en una sensación de presencia diferente, lo que llevará al usuario a percibir el mundo virtual como irreal (Sadowski y Stanney, 2002; Shneiderman, 1983; Weghorst y Billinghurst, 1993).

Para completar este apartado, y una vez revisada la bibliografía que está abordando los conceptos descritos anteriormente, se recoge a continuación una clasificación que atiende a cuatro variables: factores relacionados con la experiencia, factores relacionados con la creación del entorno virtual, factores relacionados con la tecnología (software y hardware) y factores relacionados con usuario desde el punto de vista interno, los cuales van a influir directamente en el grado de presencia y cómo esta afecta al usuario. 
En cuanto a los factores relacionados con la experiencia, en esta categoría se incluyen variables relacionadas con el nivel de control, la duración de la producción inmersiva (diferente si se trata de RV o vídeo en $360^{\circ}$, que cuenta con una duración predefinida) y el punto de vista que adopta el usuario (Witmer y Singer, 1994, 1998). Además, este último aspecto se vincula con la capacidad de este de modificar el mundo virtual al que accede o de interactuar con los elementos que aparecen en él. En relación al usuario de este tipo de contenido, sobre esta línea han trabajado autoras como Benítez y Herrera (2017) o Paíno y Rodríguez (2017); concretamente estas últimas, diferencian tres tipos de usuario:

1. Usuario observador: sólo puede moverse y cambiar el ángulo de visión, pero no puede interactuar con el entorno ni con los personajes.

2. Usuario decisor: puede tomar decisiones en momentos puntuales. Por ejemplo, puede elegir qué camino seguir o seleccionar un objeto determinado para activar una capa nueva de contenido.

3. Usuario jugador: ejerce un rol determinado y tiene un objetivo. (Paíno y Rodríguez, 2017: 11).

Respecto a los factores relacionados con la creación del entorno virtual, están vinculados al realismo de la pieza en sí misma, de los estímulos perceptuales presentados y de las posibilidades de interacción que tiene el usuario dentro de dicho entorno (Held y Durlach, 1992).

Los factores relacionados con la tecnología (software y hardware), por su parte, tienen que ver con el modo en el que se le presenta al usuario la información, desde el punto de vista técnico (Slater, Usoh y Steed, 1994; Witmer y Singer, 1994, 1998).

En último lugar, en cuanto a los factores relacionados con el usuario desde el punto de vista interno, Slater, Usoh y Steed (1994) mencionan aquellas características relacionadas con las preferencias visuales, auditivas y kinestésicas individuales y propias de cada usuario. Si bien, aunque es muy difícil influir en este tipo de factores son importantes a la hora de entender cómo afectan a la presencia.

\subsection{Periodismo deportivo en RV y vídeo en $360^{\circ}$}

Como se ha mencionado en apartados anteriores, los medios de comunicación están apostando por el periodismo inmersivo a la hora de abordar todo tipo de temáticas de interés informativo, destacando entre ellas la información deportiva. Autores como Delmazo (2019) o Sidorenko, Cantero y Herranz (2018) se han aproximado ya a su estudio. Estos últimos han realizado un repaso por las primeras producciones inmersivas que tienen al mundo del 
deporte como protagonista, principalmente desarrolladas a partir de 2016, año en el que se produce un auge importante de este tipo de piezas coincidiendo con los Juegos Olímpicos de Río de Janeiro (Sidorenko, Cantero y Herranz, 2018).

Para completar el estado de la cuestión en el presente estudio y con el propósito de ofrecer aquí una panorámica sobre los contenidos inmersivos de temática deportiva realizados en los últimos años, se ha llevado a cabo una exploración previa sobre este tipo de producciones. En este caso, se pone el foco de atención sobre tres aspectos: los productores, el tipo de deporte y la temática abordada.

Desde 2016 son muchos los medios de comunicación que han utilizado las tecnologías inmersivas para abordar temáticas deportivas; es el caso de la CNN, Euronews, Discovery Communications, HuffPost, Media VRTV, la cadena de televisión NBC, Olympic Channel, RT (Russia Today), el ente público español RTVE, Sky UK, Showtime, Sports Illustrated, The New York Times, The Sports Network, la revista Time, USA Today o el periódico surcoreano Yonhap News, entre otros. Ahora bien, es interesante apreciar que se ha observado un descenso en el número de contenidos en RV y vídeo en $360^{\circ}$ desarrollados por medios de comunicación, frente a un aumento considerable de las elaboradas por productoras audiovisuales de diversas nacionalidades, como Here Be Dragons, Jaunt, Ryot, Targo o Whitestag. También destaca la producción llevada a cabo por entidades no vinculadas al sector audiovisual, pero relacionadas con el deporte, como la Canadian Football League, la Major League Baseball, el Manchester City FC, el Aston Villa Football Club, el World Baseball Classic, el U.S. Olympic Committee o la NBA; así como de empresas relacionadas directamente, o no, con la tecnología - como Red Bull o Samsung-, e incluso realizadas por particulares.

Por otra parte, resulta especialmente significativo hacer hincapié en el tipo de deporte que se cubre desde esta perspectiva inmersiva. La razón de esto radica en que, además de los deportes más populares o que mueven mayor número de aficionados - que varían en función de cada país -, también son objeto de interés informativo otros deportes que, generalmente, tienen menor visibilidad en los medios tradicionales y que pueden ser, o no, de competición.

Entre los deportes que protagonizan las producciones inmersivas se pueden encontrar prácticas como el bobsleigh (Trending Gold: Dream Big Nigeria, Olympic Channel, 2017), la escalada (Capturing Everest. Chapter 1: The Journey, Sports Illustrated, 2017), la equitación (Get a jockey's view of the final Triple Crown race, CNN, 2018), la esgrima (En Garde! A Summer of Swords in 
New York, The New York Times, 2017), el hockey (2017 World Junior Championships-Team Canada Pre-Game Skate, Samsung Canadá, 2016), el parkour (Parkour, Vila 360, 2020), el patinaje sobre hielo (This Is Freestyle Ice Skating, Targo, 2019), el roller derby (Roller Derby 101 with the Gotham Girls of NY, NowThis360, 2017), el skateboard (Skate with pro Curren Caples, USA Today, 2017), el skydiving (Huge Group Skydive, XTreme Video, 2016), el rodeo (The 'Controlled Rage' of Bull Riding, The New York Times, 2017), el voleibol (April Ross Plays Beach Volleyball in Manhattan, The New York Times, 2017), deportes acuáticos como el kitesurfing (Kitesurfing in Buesum, Büsum Urlaub, 2019), el flyboard (Be the Iron Man by Flyboard!, VRog, 2019) o el rafting (Rafting the Rogue, 360 Labs, 2018), e incluso prácticas como el yoga (Goat Yoga, The New York Times, 2016).

Asimismo, se abordan aquellos deportes practicados por deportistas de élite y protagonizados por profesionales del baloncesto (24/8 Kobe Bryant's Final Game at Staples Center, Ueye VR, 2016), el béisbol (Blue Jays' Kevin Pillar, Samsung Canadá, 2017), el boxeo (Chris Eubank Jr., CNN, 2018) o el fútbol (360 Goal Cam: Scott Hogan, Aston Villa Football Club, 2018), entre otros; pero también por deportistas aficionados.

Si se atiende a la temática que abordan este tipo de producciones, también se pueden observar ciertos cambios. Tradicionalmente la información deportiva se ha centrado principalmente en la retransmisión de los eventos deportivos, así como en la elaboración de noticias, reportajes y entrevistas que tenían cabida en el informativo, dentro de su sección de deportes o en programas especializados.

La RV y el vídeo en $360^{\circ}$ son unas tecnologías que no están tan vinculadas a la actualidad inmediata, sino que se utilizan desde una perspectiva más atemporal. De este modo, aunque también se encuentran producciones vinculadas a la celebración de grandes eventos deportivos, como un torneo o campeonato en concreto, como la Copa Mundial de la FIFA (2018 FIFA World Cup: Mordovia Arena, RT, 2018), la Copa del Rey de baloncesto (Copa del Rey 2018 en $360^{\circ}$, Movistar Plus, 2018), la Congressional Cup de vela (Congressional Cup 2018 - Highlights, LBYC VR Media, 2018) e incluso de la celebración de una maratón popular (Shadow Runners in the N.Y.C. Marathon, The New York Times, 2016), también se identifican otras temáticas como: biografías de deportistas (Awakening: A Prologue to Nakuru Kuru, Jiva VR, 2018), la historia de un deporte, equipo, agrupación o sociedad deportiva (The Modern Games, The New York Times, 2016), entrenamientos o explicaciones sobre las reglas de un deporte en concreto (VR Demo: Strasburg's fastball-changeup combination, 
MLB, 2019), así como piezas que permiten acompañar a un determinado deportista en sus rutinas diarias (Portrait of a racing driver, Whitestag, 2019).

También aparecen producciones con un enfoque del deporte social, a través de testimonios de personas para las que constituye una parte fundamental de sus vidas. Esto es lo que sucede en Punch With Pakistani Girls at a Karachi Boxing Club (The New York Times, 2017), que cuenta cómo ha cambiado la práctica del boxeo en un pequeño club local la vida de varias jóvenes pakistaníes. Todos los ejemplos señalados anteriormente dan buena cuenta de la gran cantidad y variedad de temáticas sobre las que versan este tipo de piezas inmersivas.

\section{Metodología}

Teniendo en cuenta los aspectos señalados en el apartado teórico se describe, a continuación, el planteamiento metodológico en el que se basa la presente investigación.

\subsection{Hipótesis y objetivos}

Este estudio parte de las siguientes hipótesis:

H1. El periodismo deportivo inmersivo introduce cambios en el rol del espectador-usuario, vinculados a generar una experiencia deportiva.

H2. Desde el punto de vista narrativo, los cambios que se producen en el nuevo espectador deportivo inmersivo tienen como consecuencia un detrimento en el tratamiento informativo periodístico de dichos eventos deportivos.

Para abordar estas hipótesis se plantean los siguientes objetivos de investigación:

1. Determinar la función que cumplen los elementos narrativos vinculados al espectador.

2. Establecer cómo se produce el tratamiento de la información deportiva en las producciones inmersivas.

3. Identificar el rol que adquiere el espectador-usuario dentro de las producciones periodísticas inmersivas deportivas.

\subsection{Selección y justificación de la muestra}

Para llevar a cabo la investigación planteada se ha efectuado, en primer lugar, una búsqueda en las principales plataformas que distribuyen contenidos en $\mathrm{RV}$ y vídeo en $360^{\circ}$. Concretamente, esta ha sido realizada entre el 5 de marzo y el 5 de abril de 2020, en las stores de Oculus, Samsung VR, Viveport (de HTC) y Playstation. En todos los casos se ha filtrado previamente sus contenidos a fin de seleccionar únicamente aquellas de tipo informativo y que abordarán 
temáticas vinculadas con el deporte; por lo tanto, no han sido incluidos en la muestra los juegos y los contenidos de ficción.

Una vez realizada la búsqueda se ha seleccionado la muestra de manera aleatoria de entre el conjunto de producciones que respondieron a los criterios mencionados anteriormente. De este modo, la muestra ha quedado finalmente constituida por 225 piezas de diferente autoría y año de producción, entre 2015-2020.

\subsection{Herramientas metodológicas}

Para abordar el citado objeto de estudio se ha realizado un análisis de contenido, el cual ha contemplado una doble dimensión: cuantitativa y cualitativa. Para ello se ha elaborado una ficha de análisis que engloba cuatro aspectos fundamentales: elementos narrativos de las piezas inmersivas deportivas, punto de vista del espectador, participación del usuario y tratamiento de la información deportiva (tabla 1).

Tabla 1. Ficha de análisis

\begin{tabular}{|l|l|c|}
\hline \multicolumn{1}{|c|}{ Variable } & \multicolumn{1}{|c|}{ Indicador } & \multicolumn{1}{c|}{ Valor } \\
\hline \multirow{4}{*}{$\begin{array}{l}\text { Elementos narrativos piezas } \\
\text { inmersivas deportivas }\end{array}$} & \multicolumn{1}{|c|}{ Ubicación de la cámara } & $\begin{array}{r}\text { Tipo de distancia focal } \\
\text { Tipo de angulación }\end{array}$ \\
\cline { 2 - 3 } & Movimiento de cámara & Fija/movimiento \\
\cline { 2 - 3 } & Presencia del periodista & Sí/No \\
\cline { 2 - 3 } & Presencia de la fuente & Sí/No \\
\hline \multirow{2}{*}{ Punto de vista del espectador } & Testigo-espectador & Sí/No \\
\cline { 2 - 3 } & Protagonista & Sí/No \\
\hline \multirow{3}{*}{ Participación del usuario } & Observador & Sí/No \\
\cline { 2 - 3 } & Decisor & Sí/No \\
\cline { 2 - 3 } & Jugador & Sí/No \\
\hline \multirow{2}{*}{$\begin{array}{l}\text { Tratamiento de la información } \\
\text { deportiva }\end{array}$} & Desarrollo informativo & \multicolumn{1}{|c|}{ Sermo } \\
\cline { 2 - 3 } & Desarrollo descriptivo & \\
\hline
\end{tabular}

Fuente: elaboración propia. 


\section{Análisis de datos y resultados}

\subsection{Función que cumplen los elementos narrativos de las piezas inmersivas deportivas que afectan al espectador-usuario}

Los elementos narrativos que afectan al espectador-usuario dentro de las producciones inmersivas deportivas vienen definidos, por una parte, por 'la ubicación' y el 'movimiento de cámara'; y, por otra, por la 'presencia del periodista y de la fuente'. En relación al primer aspecto señalado, hay que recordar que cuando el usuario accede a la pieza con gafas de RV, ocupa el lugar en el que se ha situado la cámara en el momento de la grabación, por lo que una correcta ubicación de la misma repercute en cómo consume la pieza el espectador-usuario.

En el análisis de la 'ubicación de la cámara', se han tenido en cuenta dos factores intrínsecamente ligados a ella: la 'distancia focal' y la 'angulación'. La primera contempla diferentes tipos de distancias teniendo como punto de referencia el sujeto u objeto grabado. De este modo, dentro de las producciones inmersivas se identifican una distancia larga, que se podría vincular con un plano general, en términos de narrativa audiovisual; una distancia focal media, asociada a un plano americano; una corta, a un plano medio; muy corta, a un primer plano; o abstracta - esta última en aquellos casos donde la cámara se ha situado en un lugar artificial o que no permita concretar su ubicación (por ejemplo, un espacio recreado digitalmente)-. Las distancias media y corta, principalmente, van a contribuir a generar un mayor grado de presencia. Estas se producen principalmente cuando aparece el periodista o la fuente y están hablando a cámara, de manera que el espectador-usuario tiene la sensación de que le están hablando directamente a él, como si se tratara de una conversación.

Por otra parte, en relación a la 'angulación', los planos frontales que sitúan la cámara a la altura de los ojos, llevan una la visión objetiva del espectador-usuario sobre los hechos narrados; frente a las angulaciones que implican una altura por encima o debajo del sujeto y que se perciben más irreales.

Hay que especificar que cada pieza puede utilizar diferentes distancias focales y angulaciones a lo largo de la misma, a través del uso del montaje. 


\section{Gráfico 1. Distancia focal y angulación}

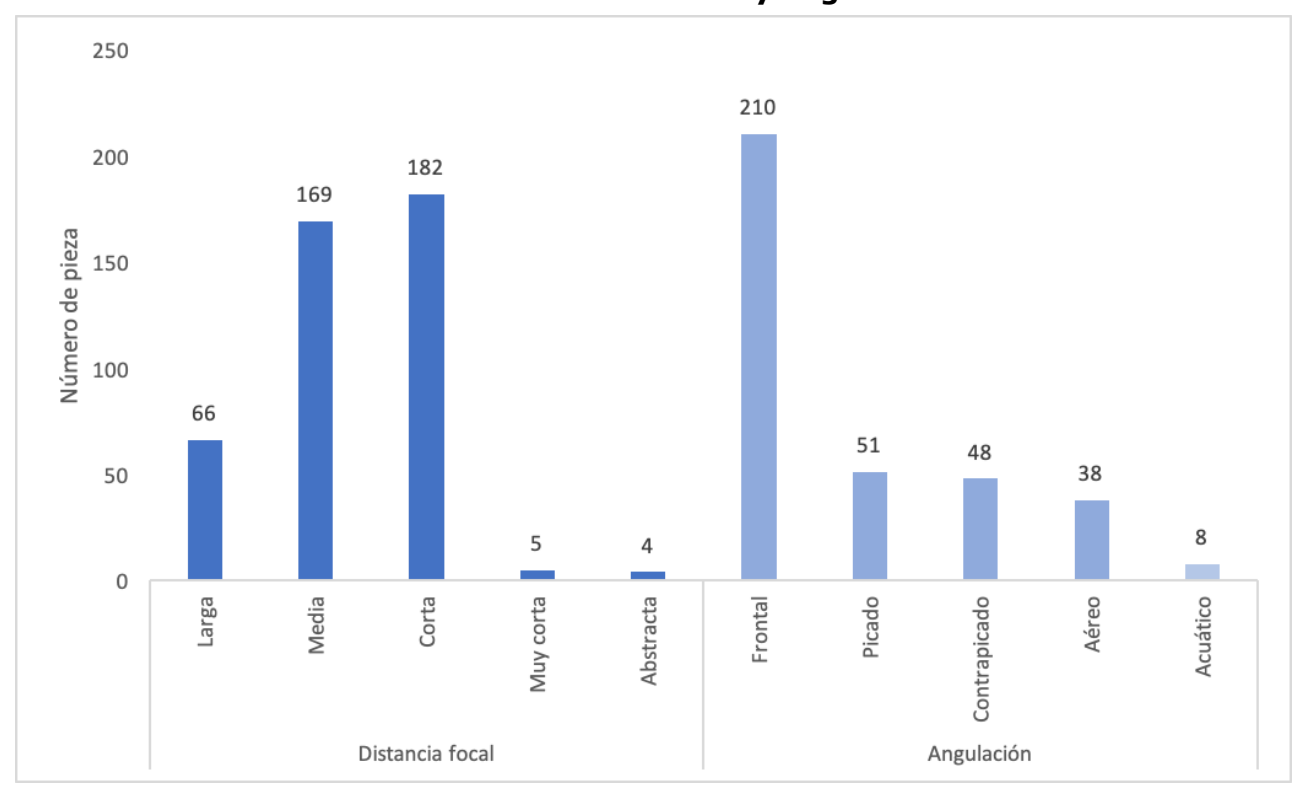

Fuente: elaboración propia.

Como se puede observar en el gráfico anterior, las distancias focales más utilizadas corresponden a las medias y cortas, y a una angulación frontal, lo que lleva a situar al espectador deportivo inmersivo en un punto de vista objetivo en relación a los hechos narrados. A modo de ejemplo, se pueden citar los casos de Trending Gold: Bring it home (Olympic Channel, 2017); The Leader Dog (Targo, 2019); Vanuatu Dreams (Samsung, 2016) o Man on Spire (The New York Times, 2016), entre otros. La distancia muy corta, como se desprende del gráfico 1, no es muy frecuente; en este caso se puede citar el ejemplo del medio surcoreano Yonhap News, titulado "지금 당장 내 차도..." 박병일 명장에 게 배우는 차량관리법 (2017), donde se sitúa la cámara en el interior del compartimento del motor de un coche. Lo mismo sucede con la distancia abstracta, presente en pocas de las producciones que han formado la muestra; un ejemplo de su utilización se encuentra en 360 Video Show Reel 2018 (360 Labs, 2019), cuando se utilizan recreaciones.

Los planos acuáticos y aéreos aparecen generalmente vinculados a deportes que se desarrollan en el agua o el aire. En el caso de los primeros, se identifican en piezas que abordan deportes como el buceo (Dolphin Man VR: Respire, ARTE, 2017), el hockey subacuático (VR 360 Underwater Hockey, Amazing Race Canada, 2018) o la natación (Vive Río, Sincronizada, RTVE, 
2016), entre otros, y que implican que la cámara tenga que sumergirse. En los planos aéreos, por su parte, puede aparecer la cámara acoplada a un dron (The Line Walkers, Targo, 2018), a algún vehículo -como un avión, helicóptero o similar- (9g with Mike Goulian-AirVenture 2016, 360 Labs, 2019) e, incluso, al propio deportista (Speed Flying 360 Video Crystal Mountain, 360 Labs, 2015). Si bien, también se han localizado planos aéreos en otras piezas relacionadas con otros deportes y que se utilizan para mostrar, por ejemplo, un estadio de fútbol desde arriba (2017 FIFA Confederations Cup: Stadium Kazan Arena, RT, 2017).

En relación al uso de los 'movimientos de cámara' en las producciones objeto de estudio, se ha observado que pueden realizarse a lo largo de toda la pieza, en momentos puntuales de la misma o, por el contrario, no contemplar ningún tipo de desplazamiento. El tipo de movimiento que se identifica es el del travelling, ya que el movimiento panorámico lo hace el espectador-usuario rotando sobre sí mismo en el momento de visionar la pieza.

\section{Gráfico 2. Movimiento de cámara}

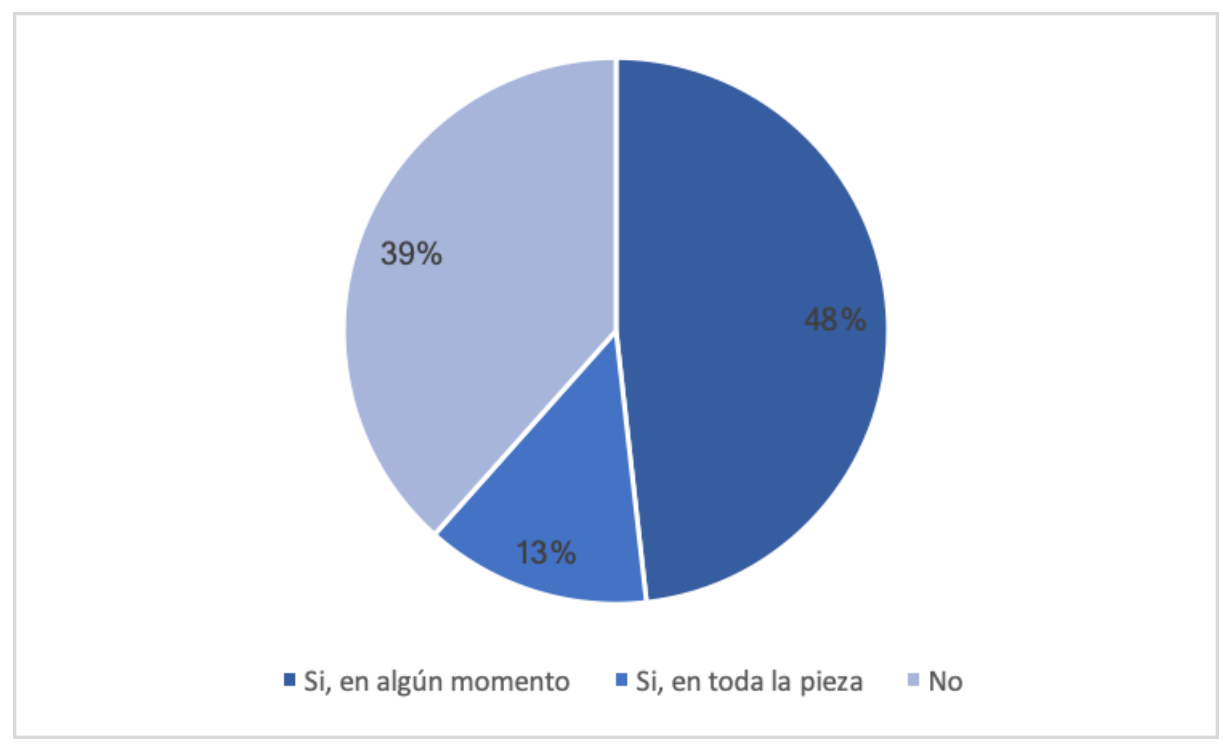

Fuente: elaboración propia.

Como se puede apreciar en el gráfico 2, los datos muestran una preferencia por desplazar la cámara, en más de la mitad de la muestra analizada. Esto es lo que ocurre en A lap at the Indy 500 with Mario Andretti (USA Today, 2016), que utiliza el movimiento en toda la pieza, o en Living the Jai Alaife (Discovery Communications, 2017), en momentos puntuales de la misma. 
Ahora bien, un porcentaje elevado de piezas opta por mantener la cámara siempre fija, como sucede en Learning on a Simulator: SSi Racing Team Documentary (Reel Again Media, 2020).

La forma en la que se realice este desplazamiento va a influir también en la recepción de la pieza por parte del usuario. No es lo mismo que la cámara se sitúe sobre el capó del coche (en la parte externa del vehículo), como sucede en Race in the Ford GT supercar (CNN, 2018), a que se ubique en el asiento vacío del copiloto (en la parte interior), como ocurre en Portrait of a racing driver (Whitestag, 2019). La primera se torna más irreal, mientras que la segunda es más verosímil. Lo mismo sucede si se trata de un avión, una moto, una bicicleta, un barco o un globo aerostático, por ejemplo. En este último caso, situar la cámara fuera de la cesta implica que el usuario también sienta que está fuera de ella, como en Hot Air Balloon Ride Bavaria (AeroFotografie, 2018).

Por último, dentro de este epígrafe se analiza la 'presencia del periodista y de la fuente'. Como muestra el gráfico 3, en las piezas deportivas inmersivas objeto de estudio hay una clara preferencia por la figura de la fuente, la cual aparece en el 62,66 \% de las producciones analizadas. Por el contrario, se puede observar un menor volumen de piezas que opten por introducir la figura del periodista; solo el 29,33\%.

\section{Gráfico 3. Intervención del periodista y la fuente}

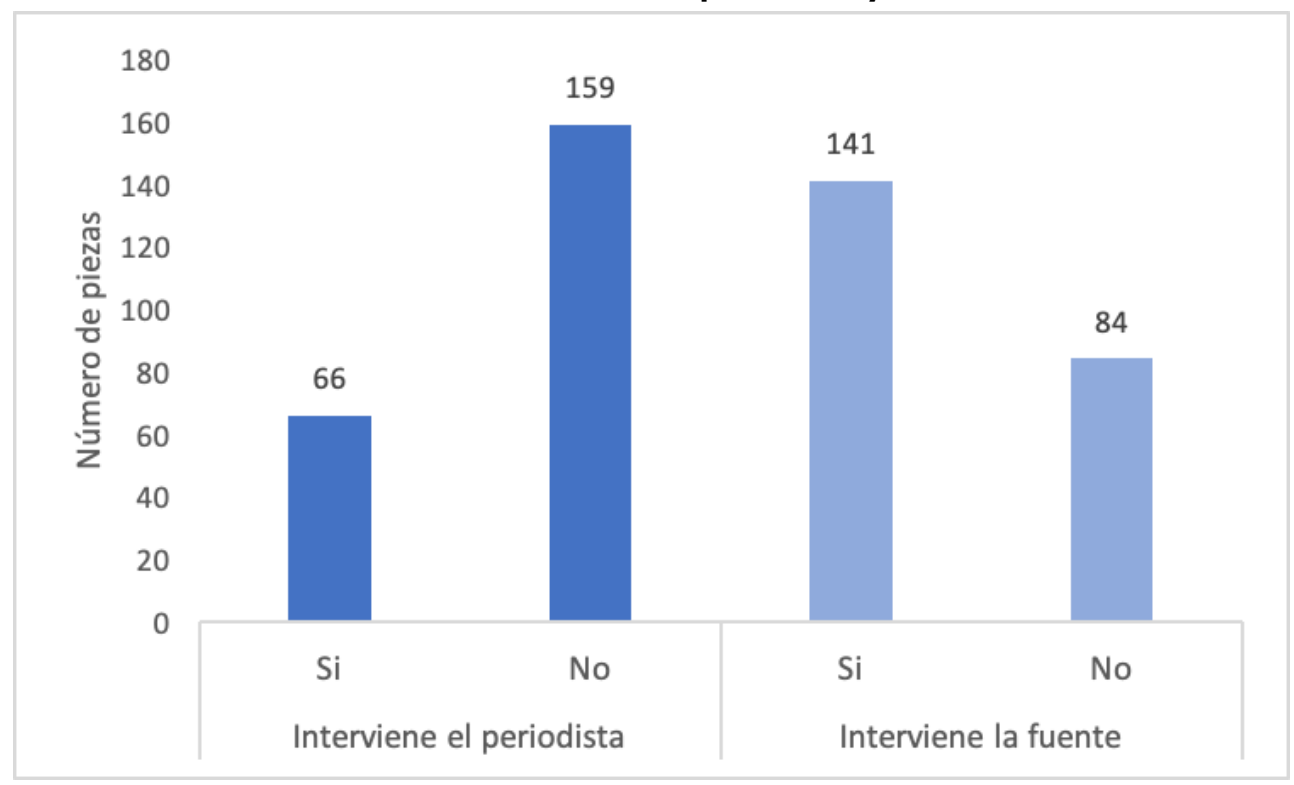

Fuente: elaboración propia. 
Cuando interviene el periodista en la pieza inmersiva deportiva, puede hacerlo de varias formas: 'físicamente y hablando a cámara' (The Birth of the Farmbird, Discovery Communications, 2017), en 'voz en off' - como narrador(24/8 Kobe Bryant's Final Game at Staples Center, Ueye VR, 2016)' o 'combinando ambas formas' (Living the Jai Alaif, Discovery Communications, 2017).

Del mismo modo, la fuente puede aparecer en las tres formas señaladas anteriormente. A este respecto, pueden mencionarse ejemplos como Houston's First World Series Parade (The New York Times, 2017), en la que la fuente habla a cámara; Track to winner's circle at the Indy 500 (USA Today, 2016), en la que aparece sólo como narrador (voz en off); o 여자축구 지소연, 모교 찾아 후배들 지도 (Yonhap News, 2017), donde aparece tanto físicamente como en off.

Asimismo, hay que especificar que en 50 de las 225 piezas analizadas aparecen tanto el periodista como la fuente. Es el caso de Skate with pro Curren Caples (USA Today, 2017) o Tour UT's Stadium like a Longhorn (Time, 2017).

\subsection{Cambios en el punto de vista del espectador en las producciones inmersivas deportivas}

Los cambios descritos anteriormente, relacionados con los elementos narrativos que intervienen en las piezas inmersivas deportivas, permiten seguir profundizando en la figura del espectador-usuario de este tipo de contenidos. La narración clásica contempla al espectador-usuario fuera de la pieza informativa, es decir, asiste a la narración de los hechos noticiosos desde la perspectiva en tercera persona. En este sentido, uno de los cambios más significativos que introduce el tipo de narración inmersiva es precisamente el cambio en el punto de vista que adopta el espectador-usuario con respecto a la información. Ahora bien, la citada inmersión puede darse a través de dos modalidades: una que contempla a un espectador-usuario como 'testigo de la información', y otra que da un paso más allá, permitiéndole ser un 'protagonista más en la historia'. La diferencia entre ambas radica en que en la primera modalidad la cámara adopta planos tanto objetivos como subjetivos, mientras que en la segunda la ubicación de la cámara ofrece un tipo de plano meramente subjetivo.

Las producciones en las que el espectador-usuario es 'testigo de la información' son las más numerosas en el conjunto de la muestra objeto de estudio; concretamente se han identificado 157 piezas que optan por este punto de vista (el 69,78 \% de la muestra). En este caso cabe señalar ejemplos como Vertical (The Vrain, 2016) o The Birth of the Farmbird (Discovery Communications, 2017). Si bien, también ha sido posible identificar un buen número de producciones donde la cámara se sitúa en un punto de vista subjetivo, bien a lo largo de toda la pieza o en momentos puntuales de la misma; esto sucede en 
68 de las producciones analizadas (el 30,22 \% de la muestra). Lo más habitual en estos casos es que la cámara aparezca acoplada al deportista, generalmente al casco, de manera que el usuario tiene la sensación de estar protagonizando - coprotagonizando - la historia. Esta perspectiva es muy común en piezas vinculadas al mundo del motor, como $360^{\circ}$ MotoGP ${ }^{T M}$ Spielberg ride (Red Bull, 2019) o Jumping Off a Plane During Memorial Day Weekend (The New York Times, 2017).

\subsection{Participación del usuario en la información deportiva inmersiva}

La totalidad de las piezas recogidas en la muestra corresponden a vídeos en $360^{\circ}$, aunque en el análisis se han identificado 8 producciones que introducen también alguna recreación tridimensional. 360 Video Show Reel 2018 (360 Labs, 2019), mencionada anteriormente, es una de las piezas que hacen uso de la recreación en momentos puntuales; no obstante, pueden mencionarse también otros ejemplos, como Urbex: Season 1 Highlights (Red Bull, 2018) o 50 Years of Special Olympics (World Games AD, 2019).

El hecho de que estas producciones opten por el vídeo en $360^{\circ}$ hace que se trate de un producto cerrado, de modo que la única posibilidad de participar que tiene el usuario es la de cambiar el ángulo de visión. Así, retomando los tipos de usuario mencionados en el apartado teórico -observador, decisor y jugador - el análisis realizado indica que la totalidad de las piezas objeto de estudio optan por un tipo de usuario 'observador' en términos de participación; es decir, no puede activar nuevas capas de información, lo que llevaría a hablar de un usuario 'decisor', ni ejercer un rol de 'jugador' como sucede, por ejemplo, en los videojuegos.

\subsection{Tratamiento de la información deportiva inmersiva}

Las figuras del periodista y de la fuente aparecen en 157 de las 225 producciones analizadas (el 69,78 \% de la muestra), lo que permite hablar principalmente de un desarrollo informativo. Estas se podrían relacionar con lo que tradicionalmente se conoce como noticias, reportajes o documentales. Bien es cierto que dicho tratamiento informativo no presenta las mismas características cuando se trata de la narración inmersiva y, en este caso, los reportajes y los documentales no son muy utilizados dentro de la información deportiva inmersiva. Prueba de ello es que, si se tiene en cuenta la duración media de las piezas objeto de estudio, esta es de 2'57"; esta duración se identificaría más con el formato de noticia reportajeada de la información audiovisual clásica.

Por otra parte, se han identificado 68 piezas (el 30,22 \% de la muestra) en las que no interviene ni el periodista ni la fuente $y$, por tanto, se concede 
completa libertad para que el espectador-usuario interprete el contenido. En estos casos puede hablarse de un desarrollo descriptivo. Esto es lo más novedoso desde el punto de vista del tratamiento de la información deportiva inmersiva, si se tiene en cuenta que en el periodismo tradicional no se contempla en ningún caso una producción donde no haya presencia desde el punto de vista narrativo del periodista y sus correspondientes fuentes.

\section{Conclusiones}

Para concluir se retoman aquí las hipótesis de las que partía esta investigación. En relación a la primera de ellas, relacionada con las transformaciones que se producen en el rol del espectador-usuario, vinculados a la creación de una experiencia deportiva, se puede afirmar que las tecnologías inmersivas han introducido cambios en relación al rol del espectador-usuario de la información audiovisual tradicional. La posibilidad de asistir en primera persona a los hechos deportivos supone la principal novedad que incorpora la narración inmersiva.

En relación al concepto de experiencia deportiva, los datos constatan que, aunque un buen número de piezas deportivas inmersivas dan un paso más allá, situando al espectador-usuario en el lugar del protagonista, esta no va a ser la tendencia habitual de manera que el mayor volumen de las producciones analizadas se va a limitar a ubicarlo como mero testigo de la información.

Resulta significativo el hecho de que la utilización de la narración inmersiva a la hora de contar los eventos deportivos introduzca la posibilidad de dar protagonismo al espectador-usuario dentro de la pieza, siendo esta una innovación con respecto a la información tradicional. Esta afirmación se refuerza si, además, se tiene en cuenta la función que cumplen los elementos narrativos inmersivos en la información deportiva.

Los resultados reflejan una clara preferencia por la utilización de distancias focales medias y cortas, y angulaciones frontales, que llevan a situar al espectador-usuario en un plano objetivo de la información deportiva. A esto se une que estas producciones también vienen caracterizadas por la preferencia del desplazamiento de cámara, ya sea a lo largo de toda la pieza o en algún momento puntual de la misma.

Ahora bien, la innovación que introducen las producciones inmersivas deportivas desde el punto de vista tecnológico no se ve reflejada en términos de participación en la narración. En este caso los resultados reflejan que dicha participación se limita a un rol de observador, de manera que no permite al usuario interactuar dentro del relato lo que, sin duda, enriquecería la experiencia dentro de la narración deportiva. 
El análisis de la presencia del periodista y de la fuente también ofrece datos reveladores vinculados a los citados cambios narrativos. El hecho de que haya una preferencia por introducir al periodista y a la fuente en las piezas inmersivas deportivas corrobora la perspectiva informativa de dichas producciones. Este aspecto permite retomar aquí la segunda de las hipótesis planteadas en este estudio que hacía referencia al posible detrimento en el tratamiento informativo periodístico de los eventos deportivos.

En relación a esta hipótesis, los resultados han mostrado que, pese a observarse una clara preferencia por el tratamiento informativo de los contenidos deportivos, es revelador el hecho de que existan piezas que no contemplan la figura del periodista y de las fuentes, ya que en la información audiovisual tradicional no existe este formato. Esto es posible gracias a las potencialidades que ofrecen las tecnologías inmersivas, aspecto que se vincula con la experiencia deportiva del espectador-usuario. En cierta medida, aunque desde el punto de vista técnico podría ser positivo, no lo es tanto si se tiene en cuenta el tratamiento periodístico que se lleva a cabo en dichas piezas, en las cuales prima una mera descripción de los hechos y la citada experiencia, en detrimento del tratamiento de la información.

Teniendo en cuenta los avances aquí descritos todo apunta a que, a medida que las tecnologías inmersivas vayan desarrollándose técnicamente, se irán produciendo nuevos avances, tanto en el rol que adquiere el espectadorusuario, como en el tratamiento que adopta este tipo de información deportiva inmersiva. Estas cuestiones dejan abiertas nuevas perspectivas de análisis sobre un objeto de estudio vivo y en constante evolución tecnológica.

\section{Referencias bibliográficas}

BENÍTEZ, M.J. y HERRERA, S. (2017). El reportaje inmersivo en vídeo 360을 diseño de un modelo de análisis. El profesional de la información, 27(1), 149-161. doi.org/10.3145/epi.2018.ene.14

BIOCCA, F. y LEVY, M. (1995). Communication in the Age of Virtual Reality. New Jersey, Estados Unidos: Lawrence Erlbaum Associates.

BRUdniY, A. y DEmilhanova, A. (2012). The virtual reality in a context of the "mirror stage". International Journal of Advances in Psychology, 1, 6-9. Recuperado de https: / / bit.ly/3hFv4kg

BUJIC, M. y HAMARI, J. (2020). Satisfaction and willingness to consume immersive journalism: experiment of differences between VR, 360 video, and article. Proceedings of the 23rd International Conference on Academic Mindtrek (AcademicMindtrek'20), 120-125.

doi.org/10.1145/3377290.3377310 
BurdeA, G. y CoIfFEt, P. (2003). Virtual Reality Technology. New Jersey, Estados Unidos: John Wiley \& Sons.

ColUSSI, J. y REIS, T.A. (2020). Periodismo inmersivo. Análisis de la narrativa en aplicaciones de realidad virtual. Revista Latina de Comunicación Social, (77), 19-32. doi.org/10.4185/RLCS-2020-1447

DE la PEÑA, N., Weil, P., Llobera, J., Giannopoulos, E., Pomés, A., SPANiAnG, B., et al. (2010). Immersive journalism: Immersive virtual reality for the firstperson experience of news. Presence: Teleoperators and virtual enviroments, XIX (4), 291-301. doi.org/10.1162/PRES_a_00005

GUBERN, R. (1996). Del bisonte a la realidad virtual: la escena y el laberinto. Barcelona: Anagrama.

HEETER, C. (1992). Being There: The Subjective Experience of Presence. Presence Teleoperators \& Virtual Environments, 1(2), 262-271. doi.org/10.1162/pres.1992.1.2.262

HERRERO, F.J. (2019). Las redes sociales como medidoras de audiencias de la prensa deportiva española. Apunts: Educación física y deportes, 135, 26-35. doi.org/10.5672/apunts.2014-0983.es.(2019/1).135.02

IDC (2019). Worldwide Spending on Augmented and Virtual Reality Expected to Reach $\$ 18.8$ Billion in 2020, According to IDC. Recuperado de https: / / bit.ly/32Aujoy

LANIER, J. (1989). An interview with Jaron Lanier. Whole Earth Review, 109118. Recuperado de https://goo.gl/tX80kn

KAnG, S., O’Brien. E., Villarreal, A., LEE, W. y Mahood, C. (2019) Immersive Journalism and Telepresence, Digital Journalism, 7(2), 294-313. doi.org/10.1080/21670811.2018.1504624

NASH, K. (2018). Virtual reality witness: exploring the ethics of mediated presence. Studies in Documentary Film, 12(2), 119-131. doi.org/10.1080/17503280.2017.1340796

NunES, A.C.B. y CANAVILHAS, J. (2020). Journalism Innovation and Its Influences in the Future of News: A European Perspective Around Google DNI Fund Initiatives. En VÁzQuEZ-HERrero, J., Direito-REbollal, S., SilvA-RodríGuEZ, A. y LóPEZ-GARCÍA, X. (eds.) Journalistic Metamorphosis. Studies in Big Data, 70, 41-56. Springer, Cham. doi.org/10.1007/978-3-030-36315-4_4

PAÍnO, A. y RodRÍGUEZ, M.I. (2017). Una apuesta por el cambio en las series de ficción. La realidad virtual como estrategia narrativa al servicio de la inmersión del espectador. Anàlisi, 0(57), 1-14. doi.org/10.5565/rev/analisi.3105

PAínO, A. y RodRíguEZ, M.I. (2019). Propuesta de "géneros periodísticos inmersivos" basados en la realidad virtual y el vídeo en $360^{\circ}$ ”. Revista Latina de Comunicación Social, 74, 1.132-1.153.

doi.org/10.4185/rlcs-2019-1375 
PÉREZ-SEIjO, S. y LóPEZ-GARCÍA, X. (2019). La ética Del Periodismo Inmersivo a Debate. Hipertext.Net, 18, 1-13. doi.org/10.31009/hipertext.net.2019.i18.01

SADOWSKI, W.J. y STANNEY, K.M. (2001). Measuring and Managing Presence in Virtual Environments. STANNEY, K.M. y MAHWAH, N.J. (eds.) Handbook of Virtual Environments Technology, 362-37. New Jersey, Estados Unidos: Lawrence Erlbaum Associates.

SALAVERRÍA, R. (2020). Explorando los medios de noticias nativos digitales. Media and Communication, 8(2), 1-4. doi.org/10.17645/mac.v8i2.3044

SANTOS, M.C. DOS (2019). Narrativas y desarrollo de contenido inmersivo: aplicación de sistemas bi y multisensoriales de realidad virtual en el periodismo. Intercom, 42(3), 133-150. doi.org/10.1590/1809-5844201937

SHERMAN, W. y CRAIG, A. (2003). Understanding virtual reality. San Francisco, Estados Unidos: Morgen Kaufman Publishers.

Shim, W. y Jounghuyum, G. (2003). Designing for Presence and Performance: The Case of the Virtual Fish Tank. Presence Teleoperators \& Virtual Environments, 12(4), 374-386.

doi.org/10.1162/105474603322391613

ShneIDERMAN, B. (1983). Direct Manipulation: A Step Beyond Programming Languages. Computer, 16(8), 57-69.

doi.org/10.1109/MC.1983.1654471

Sidorenko, P., CANTERO, J.I. y HERRAnZ, J.M. (2018). Periodismo y realidad virtual: la tecnología al servicio de la información deportiva. GoNZÁLEZESTEBAN, J.L. y GARCÍA-AVILÉS, J.A. (coords.) Metamorfosis. Radiografía de la innovación en periodismo, 137-150. Alicante: Sociedad Española de Periodística.

SLATER, M. (1999). Measuring presence: A response to the Witmer and Singer presence questionnaire. Presence: Teleoperators and Virtual Environments, 8, 560-565. doi.org/10.1162/105474699566477

Slater, M. (2003). A note on presence terminology. Presence Connect, 3(3). Recuperado de https: / / bit.ly/3cenJqX

Slater, M., LotTo, B., ARnold, M.M. y SÁnchEz-Vives, M. (2009). How we experience immersive virtual environments: the concept of presence and its measurement. Anuario de Psicología, 40(2), 193-210. Recuperado de https: / / bit.ly/3mxzzkA

SlATER, M. y SÁNCHEZ-VIVES, M.V. (2016). Enhancing Our Lives with Immersive Virtual Reality. Frontiers in Robotics and AI, 3(74), 1-47. doi.org/10.3389/frobt.2016.00074 
WEGHORST, S. y BELLINGHURST, M. (1993). Spatial perception of immersive virtual environments. Seattle, Estados Unidos: University of Washington.

WiTMER, B.G. y SinGER, M.J. (1994). Measuring Presence in Virtual Environments (ARI Technical Report 1014). Alexandria, Estados Unidos: U. S. Army Research Institute for the Behavioral and Social Sciences.

WITMER, B.G. y SinGER, M.J. (1998). Measuring Presence in Virtual Environments: A Presence Questionnaire. Presence, 7(3), 225-240. Recuperado de https: //bit.ly/33vnXG4 